\title{
La educación para el desarrollo en el nivel de posgrado: la propuesta pedagógica de la Maestría en Ciencias Sociales de la Universidad de Palermo, Argentina
}

\author{
Graciela Tonon', Damián Molgaray²
}

Resumen: En este trabajo presentaremos la propuesta pedagógica de la Maestría en Ciencias Sociales de la Universidad de Palermo, Argentina, que es un programa de posgrado que estimula en sus participantes el desarrollo de capacidades de pensamiento complejo y crítico y el compromiso con la realidad social y la diversidad humana. Esta Maestría tiene su eje en la generación de conocimientos que respondan a los problemas de la época con proyección futura; está basado en principios éticos de respeto a las personas, en tanto protagonistas, y a sus derechos; contribuyendo a la formación personal y colectiva, respetando la interculturalidad, la cooperación, la equidad y los derechos humanos. Todas estas características lo señalan como un programa que sigue los lineamientos de la educación para el desarrollo.

Palabras clave: Educación para el desarrollo, posgrado, sujetos protagonistas.

Fecha de recepción: 15 de marzo de 2020.

Fecha de admisión definitiva: I5 de junio de 2020.

\footnotetext{
' Doctora en Ciencia Política. Directora de la Maestría en Ciencias Sociales, del Centro de Investigación en Ciencias Sociales (CICS-UP) y del Journal de Ciencias Sociales de la Facultad de Ciencias Sociales de la Universidad de Palermo. Buenos Aires, República Argentina.

${ }^{2}$ Magíster en Ciencias Sociales. Docente e investigador del Centro de Investigación en Ciencias Sociales (CICS-UP) de la Facultad de Ciencias Sociales de la Universidad de Palermo. Buenos Aires, República Argentina.
} 
Development Education at postgraduate level: pedagogical proposal for the Master's in Social Sciences de la Universidad de Palermo, Argentina

Abstract: In this paper we are presenting the pedagogical proposal for the Master of Social Sciences by the University of Palermo, Argentina; a postgraduate program that stimulates in its participants the development of complex and critical thinking skills, and the commitment to social reality and human diversity. This Master's Degree is based on the principle of generating knowledge that responds to the problems of the time while creating a vision for the future; it is based on ethical principles of respect for people and their rights, making them the central focus. It contributes to personal and collective training, promotes interculturalism, cooperation, equity and human rights. All of these characteristics make it a program that reflects the guidelines and fits into the framework of development education.

Key words: Education for development, postgraduate studies, main subjects.
L'éducation pour le développement au niveau du troisième cycle universitaire: la proposition pédagogique du Master of Social Sciences de la Universidad de Palermo, Argentina

Résumé: Dans ce document, nous présenterons la proposition pédagogique du Master en Sciences Sociales, qui est un programme de troisième cycle de IUniversité de Palermo, Argentine, qui stimule chez ses participants le développement de compétences de pensée complexe et critique et l'engagement envers la réalité sociale et la diversité humaine. $\mathrm{Ce}$ Master est basé sur l'idée de la génération de connaissances qui répondent aux problèmes de l'époque en créant une projection dans l'avenir; il se fonde sur des principes éthiques de respect des personnes, en tant que protagonistes, et de leurs droits ; il contribue à la formation personnelle et collective, dans le respect del'interculturalité, de la coopération, de l'équité et des droits de l'homme. Toutes ces caractéristiques en font un programme qui suit les lignes directrices de l'éducation pour le développement.

Mots clé: Éducation au développement, études de troisième cycle, sujets protagonistes.

\section{Introducción: acerca de las Ciencias Sociales}

El debate acerca de las Ciencias Sociales no es nuevo. Ya hace más de cuatro décadas Schutz (1973) expresaba que el objetivo de las Ciencias Sociales era

lograr un conocimiento organizado de la realidad social [...] tal como lo experimenta el pensamiento de sentido común de los sujetos que viven su existencia cotidiana entre sus semejantes con quienes los vinculan múltiples relaciones de interacción (Schutz, 1973, p. 75).

Posteriormente, en la década del 90, Dogan y Pahre (1993) señalaban que el escenario internacional mostraba que no existía un consenso acerca de los 
contornos de las Ciencias Sociales y que esto generaba la existencia de distintas clasificaciones al respecto.

En el siglo XXI podemos decir que las ciencias sociales

intentan comprender nuestro mundo intersubjetivo como un universo de interpretaciones compartidas sin el cual no puede entenderse la producción y reproducción misma de la realidad social (Nuñez, 2012, p. 51).

En este caso particular, la denominación Ciencias Sociales remite a una definición amplia, no tradicional ni dogmática, que permite la evolución permanente e incluye los problemas sociales actuales y por venir, y que reconoce la innovación como proceso de aportación de lo nuevo en el campo del conocimiento científico.

La Maestría en Ciencias Sociales de la Universidad de Palermo se presenta como una propuesta pedagógica de posgrado que tiene como eje el desarrollo de capacidades de pensamiento complejo y crítico, así como su compromiso con la realidad social y la diversidad humana, trabajando desde una mirada innovadora que considera la indexicalidad (Sotolongo Codina y Delgado Díaz, 2006). Al respecto cabe aclarar que Ruiz Olabuenaga e Ispizua $(1989$, p. 56) ya habían definido este concepto diciendo que la indexicalidad es la propiedad que revela el significado contextual, mostrando la dependencia de los actos respecto del contexto situacional en el cual los mismos suceden. Asimismo, Sotolongo Codina y Delgado Díaz (2006, p. 90) expresaron que el conocimiento en Ciencias Sociales tiene que estar apegado a los contextos de realidad donde tienen lugar los acontecimientos que modulan las relaciones y el devenir de los procesos sociales.

En la actualidad el debate acerca de las Ciencias Sociales persiste, y lo que queda claro es que más allá de los cambios y de las distintas opiniones, las Ciencias Sociales contribuyen al desarrollo de las sociedades y tienden a posibilitar la definición de cambios en las políticas para facilitar la participación ciudadana, potenciando la democratización del sistema político.

Así pues, en este trabajo se analizarán las particularidades de la Maestría en Ciencias Sociales de la Universidad de Palermo (Argentina) que la identifican como una carrera de posgrado que sigue los lineamientos de la educación para el desarrollo. No se abordará una experiencia en particular, sino el despliegue de la propuesta de esta carrera que lleva ya tres años de existencia. Para eso, primero se hará un breve repaso sobre el rol que asumen las universidades en el siglo XXI, especialmente en regiones como la sudamericana; seguidamente se aportarán algunas claves teóricas sobre la educación de posgrado, precisando en 
el enfoque de la educación para el desarrollo. Luego, el texto se adentrará en la estrategia pedagógica de la Maestría para concluir con algunas reflexiones finales.

\section{El rol de las universidades en el siglo $X X I$}

Si se interrogara sobre el rol actual que le cabe a la universidad contemporánea en las sociedades democráticas sudamericanas en el siglo XXI, la respuesta conllevaría a reflexionar sobre las particularidades de las sociedades actuales. Influidas por la globalización, signadas por la incertidumbre global y afectadas por la sensación de riesgo cotidiano, la representación colectiva de la educación y, particularmente, de la vida universitaria, estas sociedades parecen convertirse en un nuevo espacio de participación, encuentro, refugio y arena de múltiples desafíos.

Altbach (2009), rescata el importante papel que afrontan las universidades en las sociedades democráticas, especialmente por su tendencia a liderar debates nacionales, tanto de cuestiones académicas, así como también de temas propios de la actualidad local y nacional de los países. En este sentido, el mismo autor subraya la atmosfera intelectual abierta y la promoción de nobles valores como fenómenos subsidiarios de los procesos de construcción y consolidación democrática: entendiendo por democracia aquél régimen político que tiene la capacidad de enriquecer el encuentro razonado, viabilizando discusiones interactivas en donde pueden ser escuchadas voces diferentes y con procedencias distintas (Sen, 2011).

Así, entonces, podría decirse que las universidades se han convertido en instituciones sociales íntimamente involucradas en las diversas áreas de la vida pública de los ciudadanos, con compromisos explícitos frente a las necesidades de la sociedad. Con esto, es importante subrayar el elemento de confianza que recae sobre ellas, convirtiéndolas en un espacio de potencial construcción de ciudadanía. Ciudadanía que, vale decir, ya no podría enunciarse desde su definición clásica o moderna, dado que presenta -hoy- características más contemporáneas, signadas por la presencia de nuevos nichos de identificación de los sujetos. En términos generales, la noción de ciudadanía contempla todas aquellas dinámicas sociales en las que se delinea cierto entramado simbólico de referencia, a través del cual los sujetos se identifican con alguna comunidad de pares, desarrollando diversos tipos de participación que implican, desde el decir de Cortina (2011, p. 98), una especie de "ser con otros".

A finales del siglo XX, Lechner (1990, pp. 44 y 45), ya advertía la manera en que las rasgaduras de las identidades colectivas tendían a generar nuevas re- 
composiciones al margen de las instituciones sociales existentes; decayendo los ámbitos de sociabilidad informal. En esa misma oportunidad, Lechner (1990) se preguntaba acerca de qué "faros" podrían ayudar a iluminar el mundo de las vidas individuales y cotidianas de los sujetos, en función de la vida colectiva en la cual les tocaba transitar. Como reflexión, entonces, valdría la pena pensar hoy cómo, la adaptación progresiva de los modos de conocimiento producidos en la universidad-adecuados a formatos más colaborativos y a la vez más disruptivos de las tradicionales prácticas coloniales del saber- originan formas de integrar nuevos (y antiguos) modos de conocer y de interpretar la realidad.

Las instituciones universitarias se encuentran hoy frente al desafío de viabilizar la producción y difusión de saberes desde lógicas localistas, que se integren a los diálogos globales desde una remozada narración que se atreva también a definir un nuevo tipo de identidad social: atenta a la diversidad cultural como fuente de creatividad, innovación y equidad social.

La participación amplia e inclusiva en la educación superior, según Reiseberg y Watson (2011), se ha erigido como un elemento clave del imaginario ciudadano en términos de justicia social e igualdad de oportunidades, así como también de reconocimiento social.

\section{La educación de posgrado enfocada al desarrollo}

Considerando la descripción desarrollada en el apartado anterior acerca de las universidades en el siglo XXI, cabe en este momento avanzar en torno a la definición de la educación de posgrado.

Para definir la educación de posgrado es útil recordar la propuesta de Manzo Rodríguez, Rivera Michelena y Rodríguez Orozco (2006) cuando afirmaban que

... es un conjunto de procesos de enseñanza-aprendizaje dirigidos a garantizar la preparación de los graduados universitarios para complementar, actualizar y profundizar en los conocimientos y habilidades que poseen (Manzo Rodríguez et al, 2006, p. 1).

Los mismos autores mencionan que entre las funciones de la Educación de Posgrado se resumen: (a) la actualización, que posibilita la renovación sistemática de los conocimientos y habilidades profesionales a nivel de los avances y el desarrollo científico; (b) la complementación, que viabiliza la adquisición de nuevos conocimientos y habilidades profesionales no recibidos en sus estudios precedentes 0 
adquiridos sin la profundización requerida, y (c) la profundización que facilita la obtención de un nivel superior en los conocimientos científico-técnicos y habilidades profesionales en el campo específico de una profesión o de un área concreta de la ciencia y la técnica.

Asimismo, se pueden reconocer los denominados aspectos esenciales de la educación de posgrado, referidos a: la pertinencia social, la actualización y la democratización (Manzo Rodríguez et al, 2006). La pertinencia social es la que concreta el vínculo real de toda concepción posgraduada con problemas nacionales o regionales relevantes; la actualización permite que los estudios de posgrado se vinculen -con un criterio de nacionalización de sus recursos- al desarrollo científico técnico nacional e internacional, y la democratización es la que permite que este sistema educacional se auto-regule a través de la participación activa de sus integrantes (Manzo Rodríguez et al, 2006).

Desde los inicios del siglo XXI, autores como Duderstadt (2010, p. 413) señalaron que ha comenzado una nueva era en la que el conocimiento no solo es necesario para el bienestar social, sino que también es clave para mejorar la calidad de vida de cada persona. Es en este sentido que, tanto la educación como las habilidades de cada sujeto, son consideradas cada vez más asiduamente como las claves de la calidad de la vida personal y de la sociedad en general (Duderstadt, 2010, p. 425). Es por eso que la educación de posgrado, considerada como trayecto educativo de formación y perfeccionamiento que se desarrolla posterior al egreso de los estudios de grado, se ubica en un rol protagónico en las sociedades de este siglo.

Ahora bien, para relacionar la educación de posgrado con el desarrollo, tomaremos la propuesta teórica del Premio Nobel Amartya Sen (1998) quien concibe el desarrollo como un proceso de expansión de las libertades y considera a la participación como una parte constitutiva del mismo. El citado autor (1998) nos alerta acerca de que

aún si se acepta que la consecución de una mayor prosperidad económica es el eje central de los procesos de desarrollo, no se puede olvidar que este supuesto se basa en los valores compartidos por la sociedad (Sen, 1998, p. 92).

En esta línea de pensamiento la educación - así como la atención de la salud-para Sen (1998, p. 81) está más allá de la productividad económica y el bienestar inmediato, ya que el autor reconoce que la formación escolar es vital para el proceso de desarrollo y tiene efectos potenciales sobre el bienestar y las libertades de las personas durante toda su vida (1998, p. 82). 
En esta misma línea, también Nussbaum (2012) considera que la educación es valiosa en sí misma, y es una fuente de satisfacción que se mantiene constante en el ciclo vital. La autora explica que la educación ejerce una función central para el desarrollo y para la ejercitación de otras muchas capacidades humanas, razón por la cual ella la denomina como "funcionamiento fértil", subrayando así todo aquel tipo de funcionamiento que tiende a favorecer-también-a otras capacidades concomitantes (Nussbaum, 2012, p. 64).

Cabe recordar que la educación para el desarrollo surgió ligada a las prácticas de cooperación internacional y sus propuestas tanto teóricas como prácticas han ido evolucionando de forma paralela al avance del debate en torno al desarrollo, proponiendo un proceso educativo encaminado a generar conciencia crítica sobre la realidad mundial, facilitando herramientas para la participación y la transformación social en claves de justicia y solidaridad (Celorio y López de Munain, 2007, p. 124).

En este sentido, continuando con los aportes de Sen (2002) valdría decir que la equidad en la educación se vuelve también un factor ineludible de la justicia que subyace a los acuerdos sociales básicos y generales de una sociedad; pues la equidad en la realización (es decir, en las posibilidades de logro), junto a la distribución equitativa de los servicios educativos, son dos piezas fundamentales para complejizar la visión de la igualdad de oportunidades, resaltando, pues, las justas y medidas facilidades sociales de las que dispone cada grupo social en particular para acceder o garantizar su servicio educativo.

La educación para el desarrollo se basa en la persona como centro de la acción educativa y como protagonista de los procesos de enseñanza-aprendizaje; la interculturalidad como propuesta de convivencia y como marco de aprendizaje y cambio cultural; la justicia social, la cooperación, la equidad y los derechos humanos como valores para una nueva ética social y la idea de la educación como herramienta emancipadora, en términos de Freire (Celorio y López de Munain, 2007).

La Organización de las Naciones Unidas para la Educación, la Ciencia y la Cultura (UNESCO), en su rol de centro y tribuna para la reflexión intelectual y el debate sobre los nuevos planteamientos de la política y la administración de la educación, plateó claramente (desde 2015) la necesidad de que los sistemas de enseñanza del mundo avancen en una visión humanista de la educación que permita sostener a la humanidad y a su bienestar común. La educación para el desarrollo, entonces, es aquel enfoque pedagógico orientado a contribuir en la elaboración de nuevos modelos de desarrollo, en donde se integren las dimensiones múltiples de la 
existencia humana para responder eficazmente a los nuevos y diversos desafíos que impone la realidad contemporánea (como por ejemplo: la irrupción de renovados circuitos de producción y de trabajo, las crisis naturales provocadas por el vertiginoso cambio climático, la profundización de las desigualdades sociales, el desconocimiento a la diversidad cultural y la emergencia de fenómenos chauvinistas, las crisis de refugiados por migraciones masivas, entre otros); valorando los diversos sistemas de conocimientos disponibles entre las culturas del planeta y reconociendo el avance de las fronteras de la ciencia y de la tecnología, sobre todo en materia de nuevos dispositivos y aplicaciones digitales.

En un mismo sentido, la UNESCO (2015) advierte que una real educación orientada al desarrollo debe ir más allá de la mera "distribución" de competencias básicas y compartimentadas que alientan a repensar los entornos de aprendizaje, apostando por enfoques pedagógicos multidisciplinarios que contribuyan a forjar en los y las estudiantes contenidos referidos a las dimensiones sociales, económicas y medioambientales que impactan en el desarrollo sostenible de los pueblos. Trabajar en este segundo enfoque será la clave para que la educación pueda convertirse en una herramienta útil desde la cual aprender a vivir en un mundo cada vez más dinámico y complejo.

\section{La estrategia pedagógica de la Maestría en Ciencias Sociales}

La Maestría en Ciencias Sociales de la Universidad de Palermo se presenta como una propuesta pedagógica de posgrado innovadora que incluye lineamientos típicos de una iniciativa para el desarrollo. El diseño curricular de esta carrera intenta provocar una actitud crítica y comprometida con la realidad que no se restringe solamente a lo formal, sino que irradia en compromiso y corresponsabilidad actuante, fomentando actitudes y valores ciudadanos entre sus participantes.

El eje fundamental de la Maestría en Ciencias Sociales es el estudio y la generación de un conocimiento que sirva para dar respuesta a los problemas de la época, así como a la proyección futura de los mismos. En este sentido, ocupan un lugar destacado los principios éticos basados en el respeto a las personas y a sus derechos humanos. Y al hablar de derechos humanos vale rescatar las apreciaciones de Lechner (1983/2013) cuando se refería, en un juego de palabras, al derecho de las personas a tener derechos. De esta manera, los derechos humanos representan ese stock de garantías universales sin el cual el resto de derechos que provee una sociedad democrática no podrían ser efectivamente realizables 
Los diversos cursos de la Maestría -tanto obligatorios como optativos- han sido pensados desde una mirada innovadora, que respeta e integra las ideas base de las Ciencias Sociales, al tiempo que avanza sobre nuevos desafíos intelectuales. De esta manera incorpora la reflexión sobre temas teóricos que surgen de los problemas que tienen lugar en esta etapa histórica y se proyectan sobre la integración de nuevos campos temáticos, a saber: el desarrollo humano, la perspectiva de género, la interculturalidad, la calidad de vida, la seguridad, la sustentabilidad, la paz sustentable, las desigualdades, las poblaciones en movimiento, los métodos mixtos de investigación, entre otros. Se trata de un programa interdisciplinario, y en este sentido -siguiendo a Ortiz (2004, p. 144)- podría decirse que

pensar las relaciones sociales insertas en una totalidad significa decir que las fronteras entre las disciplinas no pueden ser rígidas, pues lo contrario implicaría el fraccionamiento de su comprensión.

La propuesta de la Maestría tiene como una de sus metas recuperar y combinar las especificidades/particularidades de las distintas disciplinas que conforman el campo de las denominadas Ciencias Sociales. Y es por eso que propone un trabajo interdisciplinario, al decir de Dogan y Pahre (1993), cuando expresan que la interdisciplina supera los límites de cada disciplina generando desplazamientos que sirven de base para los intercambios de métodos, conceptos y teoría.

Acerca de las competencias transversales que propone el Programa de la Maestría en Ciencias Sociales, comenzaremos por recordar en principio a Perrenoud (2004) cuando define competencia como la aptitud para enfrentar eficazmente una familia de situaciones análogas, movilizando de manera rápida, pertinente y creativa, múltiples recursos cognitivos: saberes, capacidades, micro-competencias, informaciones, valores, actitudes, esquemas de percepción, de evaluación y de razonamiento. También Perrenoud (2008, p. 1), con posterioridad, señalaba que, si bien el enfoque por competencias surgió como una tentativa de modernizar el currículum y adaptarlo a la realidad contemporánea en los campos del trabajo, de la ciudadanía o de la vida cotidiana; en el campo educativo también vincula los saberes con las prácticas sociales, las situaciones complejas, los problemas y los proyectos. Entonces trabajar por competencias implica entender el aprendizaje como un circuito multidireccional en el cual el/la estudiante tiene un rol activo que estimula la capacidad crítica, ética, creativa y sensible en la gestión de su aprendizaje para lograr una formación integral y el/la profesor/a es un/a guía que lo/a acompaña en ese recorrido (Alsina et al, 2011, p. 15).

En el campo educativo, se identifican las denominadas competencias transversales que son las que atraviesan varias disciplinas, es decir aquellas que no tienen una 
asignatura propia, sino que se van adquiriendo a lo largo de todas las asignaturas. Para Corominas Rovira (2001) las competencias transversales son aquellas que se relacionan con aprender método, relaciones sociales, trabajo en equipo, comunicación oral y escrita y capacidad de liderazgo. Para Perrenoud (2001, p. 10) son las que atraviesan varios sectores de la existencia humana, sin referencia a las disciplinas científicas ni educativas. Además, ellas son responsabilidad de todos los/as docentes más allá del curso que enseñen, requiriendo de una planificación por parte de cada programa educativo (Miro, 2011, p. 102).

En el cuadro a continuación, se muestran las competencias transversales que propone el programa de Maestría

\section{TABLA I. Competencias Transversales}

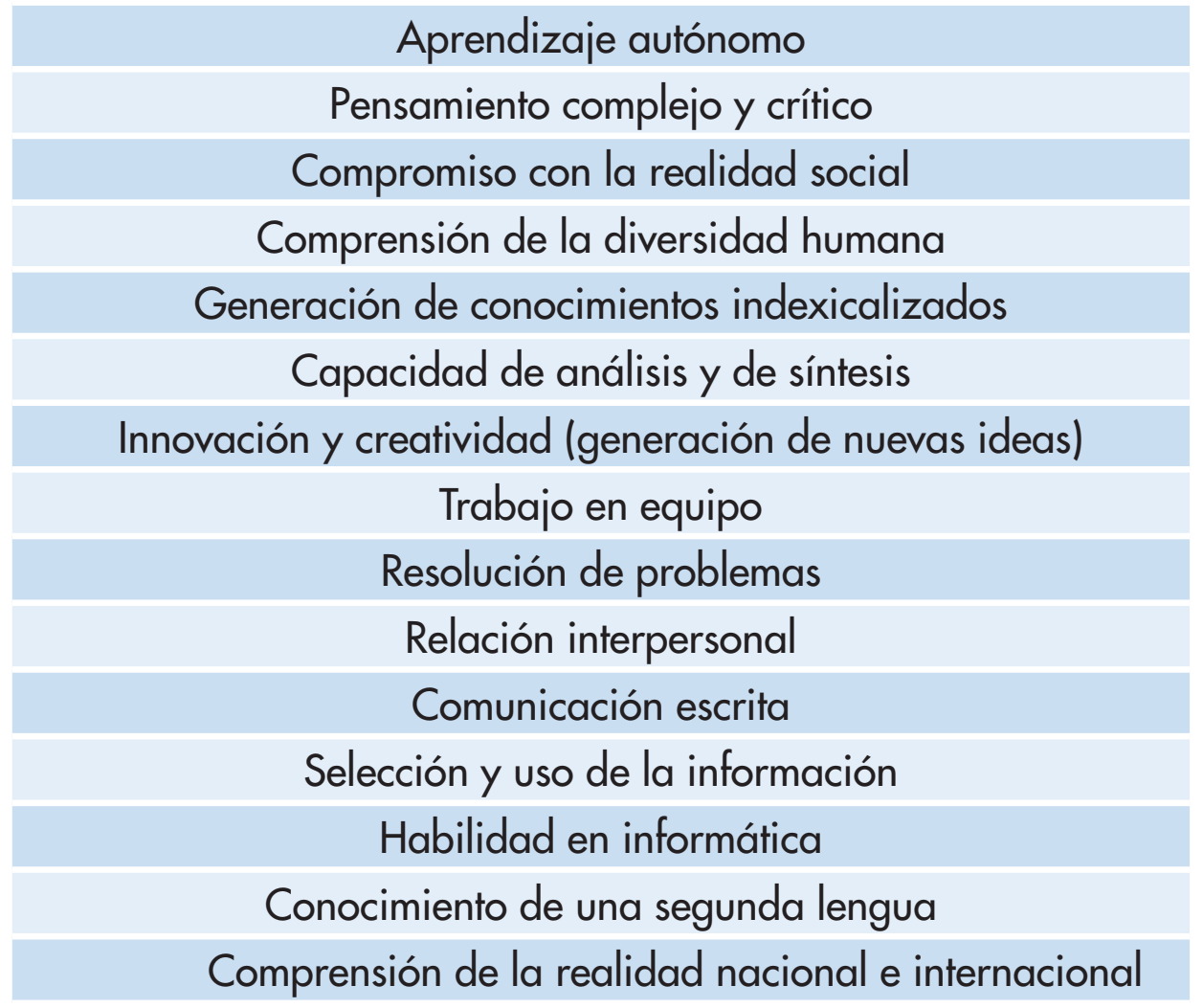

Fuente: elaboración propia de los autores.

Este programa potencia en sus participantes el desarrollo de pensamientos complejos y críticos, así como el compromiso con la realidad social y la diversidad humana, a partir de la profundización del conocimiento social en sus dimensiones teórica 
y metodológica, apostando a una práctica especifica en investigación social con posibilidad de desarrollo en el marco que ofrece el Centro de Investigación en Ciencias Sociales (CICS-UP), y con alternativas de difusión tanto en los libros que conforman la Colección de Ciencias Sociales, como en la revista científica electrónica titulada: Journal de Ciencias Sociales, ambas publicaciones de la Facultad de Ciencias Sociales de la Universidad de Palermo.

En este punto, es importante mencionar los espacios dentro de proyectos de investigación radicados en el Centro de Investigación que se reservan para los/as estudiantes de la Maestría que se interesen por temáticas singulares, como por ejemplo la dedicada al bienestar de niños y niñas o aquella focalizada en los debates sociales contemporáneos. La disponibilidad de líneas de investigación tales como las anteriormente mencionadas, revisten un ejemplo más de iniciativas típicas de la educación para el desarrollo, pues revalorizan el estudio de grupos tradicionalmente excluidos o invisibilizados.

Frente a una sociedad ciertamente dinámica y en transición, el programa de la Maestría, siguiendo a Freire (1974), apunta a evitar posiciones quietistas y, por el contrario, insta a asumir las potencialidades del cambio considerándolo como una característica esencial de las comunidades humanas y de la vida democrática. En este sentido, por ejemplo, la Maestría en Ciencias Sociales hace uso de las nuevas tecnologías de la información y de la comunicación, organizando webinars y teleconferencias para acercar a los estudiantes las experiencias y los aprendizajes de académicos residentes en distintas partes del planeta para complementar la educación formal que ofrece la carrea. Es decir, este programa ofrece posibilidades concretas para un desarrollo en investigación actualizada, con difusión internacional y con participación en redes globales de investigación.

Dado que la Maestría, por otro lado, culmina con la presentación de una tesis de investigación, que tiene como requisito ser una producción original, desde la cual aportar una innovación en el campo de las Ciencias Sociales, las posibilidades de investigación que ofrece la carrera tienen como eje de sustentación el rol protagónico de los sujetos (el sujeto investigado y el sujeto investigador) en sus contextos espacio-temporales. Esto implica el interés por las distintas relaciones que se generan y desarrollan, precisamente entre las personas, los tiempos y los lugares en los cuales viven su vida. De esta manera se recuperan sus vivencias, sus experiencias y sus opiniones.

Desde la Maestría, entonces, se propone un enfoque pedagógico fundado en la investigación, caracterizado por una estrategia en la que "se hacen visibles las 
interacciones, las herramientas didácticas o dispositivos utilizados, la organización del tiempo-espacio y las mediaciones en ellos, y propone una ruta por seguir con los pasos detallados para lograr resultados específicos de aprendizaje" (Mejía Jiménez y Manjarrés, 2011 , p. 139). Este enfoque programático y singular entre el conjunto de maestrías ofertadas en el sistema argentino, se basa en la libertad epistemológica y la diversidad metodológica, y tiene como eje la elaboración de preguntas de investigación que conlleven a la formulación del problema de investigación basados en la idea de que "la pregunta se convierte en mediación que permite poner en marcha el proceso de aprendizaje" (Mejía Jiménez y Manjarrés, 2011, p. 162). De esta manera, la Maestría intenta ofrecer medios para que sus participantes piensen auténticamente, logren una auto-reinvención y activen un real pensamiento crítico orientado al desarrollo.

Cabe en este punto recordar que son los conocimientos previos que porta cada sujeto los que hacen posible la incorporación de nuevos conocimientos, a partir del descubrimiento y la ampliación progresiva de los saberes innatos. Se generan así nuevas formas de interdisciplinariedad que permiten entender la cotidianidad de otra manera, sirviendo -justamente- como un punto de partida desde donde desplegar nuevas formas de enunciación del conocimiento a través de la investigación (Mejía Jiménez y Manjarrés, 2011, p. 159).

En este sentido, debido a la variopinta procedencias de estudiantes y profesores extranjeros que participan de la Maestría, el despliegue de la carrera se erige como una experiencia cotidiana de interculturalidad, donde el respeto a la diversidad y el enriquecimiento mutuo se forja en los mismos entornos áulicos, favoreciendo en todo momento el diálogo, la concertación y, con ello, la integración y convivencia enriquecida entre culturas y valores, que muestran una estrategia orientada al desarrollo.

También es importante señalar que, en la investigación en Ciencias Sociales contemporánea, resulta necesario tener en cuenta el saber que proviene de la práctica, tal como lo expresaron Dogan y Pahre (1993) al reconocer que numerosos conceptos provienen de ella, constituyendo, de esta manera, una respuesta a la aparición de fenómenos emergentes en la sociedad. Acerca de la relación teoría-práctica, resulta interesante señalar que la agenda de desarrollo sostenible para el año 2030, lanzada desde las Naciones Unidas (2015), apuesta a que estudiantes de todo el planeta adquieran los conocimientos teóricos y prácticos necesarios para promover un efectivo desarrollo sostenible, mediante -entre otras cosas- una educación que promueva la cultura de la paz y la no violencia, la ciudadanía mundial y la valoración de la diversidad cultural. En línea con esta idea, la Maestría invierte 
en la germinación del pensamiento complejo y en el compromiso con la realidad social y la diversidad humana; no como una entelequia, sino como un trabajo sistemático y cotidiano. El diseño de la Maestría en Ciencias Sociales tiene en cuenta lo señalado por Freire (1974) acerca de no confundir la reflexión teórica con la simple verbosidad, pues la teoría es un contacto analítico sopesado sobre la realidad para vivirla y comprobarla plenamente. Teorizar, en tanto principio y fin de la investigación científica, implica contemplar y desarrollar una capacidad de abstracción, un apego tanto a la innovación como -fundamentalmente- al estudio comprometido con la realidad social.

\section{Reflexiones finales}

En este trabajo se presentó la propuesta pedagógica de la Maestría en Ciencias Sociales de la Universidad de Palermo (Argentina), en tanto programa de posgrado que estimula en sus participantes el desarrollo de capacidades de pensamiento complejo y crítico, y que alienta el compromiso con la realidad social y la diversidad humana.

Se trata de un programa académico centrado en la teoría y en la investigación, con la característica fundamental de considerar ambas dimensiones en un contexto de desarrollo, lo que implica una reflexión profunda acerca del tiempo y del espacio en el cual este programa va tomando forma; proponiendo una visión holística de la realidad, en donde asumen un lugar protagónico los sujetos: es decir, tanto los/ as estudiantes como los/as docentes.

Los objetivos de la Maestría son: brindar una visión general y actualizada de las bases conceptuales de las Ciencias Sociales, así como de los problemas sociales de la época, tanto a nivel nacional como regional e internacional, generando en los participantes una actitud sostenida por descubrir, comprender y explicar las situaciones de vida de las personas, los grupos y las sociedades, a partir de una exploración crítica y transversal de los temas sociales. En línea con lo que advertía Lechner (1991/2015), la contribución más importante de las ciencias sociales radica en la progresiva generación de un renovado marco interpretativo de la realidad social de los pueblos para calibrar sus mapas cognitivos y así proveer una visión convergente del desarrollo que permita, entre otras cosas, la consolidación de la institucionalidad democrática.

Habiendo distinguido, en primer lugar, las potencialidades del estudio de las Ciencias Sociales y, en segundo lugar, habiendo precisado un marco descriptivo 
sobre la complejización de los roles de las universidades sudamericanas, se identificaron diversos componentes que permiten señalar al programa de la Maestría en Ciencias Sociales como un programa que sigue los lineamientos de la educación para el desarrollo, en línea con el carácter dinámico de la misma, fundada en la necesidad permanente de adaptación a los cambios surgidos en el contexto internacional, así como del impacto de estos, en el ámbito de las relaciones sociales y de las funciones asignadas a la Educación en cada momento (Celorio y López de Munain, 2007, p. 124).

Si se considera el desarrollo como un compromiso trascendental con las posibilidades de libertad (Sen, 2000), podría decirse que el programa de la Maestría en Ciencias Sociales de la Universidad de Palermo es un programa de formación de posgrado que, desde su inicio, se ha comprometido con el despliegue de las capacidades de sus participantes, proveyendo un diseño pedagógico original y comprometido con los desafíos de su tiempo, apostando por una (re)lectura innovadora de la realidad social que inspire en sus participantes una actitud ávida por nuevos enigmas de indagación orientados al desarrollo de las sociedades.

La propuesta de esta carrera de posgrado resulta, entonces, concordante con los principios de la educación para el desarrollo, dado que se basa en la persona como protagonista de los procesos de enseñanza-aprendizaje, la interculturalidad, la justicia social, la cooperación, la equidad, los derechos humanos, la ética social y la educación como herramienta emancipadora, porque:

a. Sus cursos integran las ideas básicas de las Ciencias Sociales incorporando reflexiones sobre temas teóricos que surgen de los problemas emergentes de la realidad contemporánea, apostando a una estrategia interdisciplinaria en la que se integran saberes de campos diversos y tradicionalmente ajenos a las disciplinas sociales como el arte, la literatura o las letras.

b. Ofrece los medios para que sus participantes piensen auténticamente, logren una auto-reinvención y activen un real pensamiento crítico, acorde con los desafíos inherentes a un mundo complejo.

c. Hace uso de las nuevas tecnologías para complementar la educación formal de sus estudiantes, acercando las experiencias y los aprendizajes de académicos residentes en distintas partes del planeta, abocados a realidades sociales y culturales bien diversas y que participan en redes globales de investigación. 
d. Las propuestas de investigación que se ofrecen ponen foco en el rol protagónico de los sujetos, especialmente de aquellos que se hallan en situaciones de exclusión o invisibilidad.

e. El clima áulico se basa en un espacio de interculturalidad, donde el respeto, la diversidad y el enriquecimiento mutuo se forja a partir de las distintas procedencias de sus estudiantes y profesores.

En añadidura a lo anterior, la ágil y personalizada gestión administrativa que ofrece la Universidad, con eje en las personas, sumado a las vinculaciones que se brindan desde los diversos nodos académicos con los que cuenta la Facultad de Ciencias Sociales - sin mencionar lo agradable que resultan los entornos edilicios donde se desarrollan las clases con espacios áulicos bien aclimatados y tecnológicamente equipados para la experiencia que propone el diseño de la Maestría- vuelven a la propuesta de esta carrera una iniciativa con orientación al desarrollo.

Las particularidades de este programa de posgrado motivaron su difusión en este trabajo, esperando que su progresivo crecimiento pueda continuar siendo motivo de análisis en el futuro.

Agradecimientos: Al Sr. Rector de la Universidad de Palermo, Ing. Ricardo Popovsky, a la Sra. Decana de la Facultad de Ciencias Sociales, Mag. Meds Elsa Zingman, al Sr. Vice-Rector, Mag. Matías Popovsky y al Sr. Secretario Académico de la Facultad de Ciencias Sociales Lic. Luis Brajterman.

\section{Referencias bibliográficas}

Alsina, J. (Coord.), Boix, R., Burset, S., Buscà, F., Colomina, R., García, M.A., Mauri, T., PujolÀ, J., SAYÓs, R. (2011). "Evaluación por competencias en la universidad: las competencias transversales". Cuadernos de docencia universitaria 18. Barcelona. Ediciones Octaedro. Recuperado de http://diposit.ub.edu/dspace/bitstream/24 45/145000/1/18cuaderno.pdf

AltBACH, P. G. (2009). Educación superior comparada: el conocimiento, la universidad y el desarrollo. Cátedra UNESCO-ONU "Historia y futuro de la Universidad", Colección de Educación Superior. Buenos Aires: Universidad de Palermo. 
COROMINAS ROVIRA, E. (2001). "Competencias genéricas en la formación universitaria". Revista de Educación (325), 299-321. Recuperado de hitps://pdfs.semanticscholar. org/c6c7/af71c95b0e66f10057d49a3d2d84d06bfb9a.pdf

CORTINA, A. (2011). "Los valores de una ciudadanía activa". En B. TORO y A. TALLONE (Coord.), Educación, valores y ciudadanía (pp. 95-107). Madrid: Organización de Estados Iberoamericanos para la educación, la ciencia y la cultura. Fundación SM.

Dogan, M. y PAHRE, R. (1993). Las nuevas ciencias sociales: la marginalidad creadora. México: Grijalbo.

DUDERSTADT, J. (2010). Una universidad para el siglo XXI. Tomo 2. Cátedra UNESCOONU "Historia y futuro de la Universidad", Colección Educación Superior. Buenos Aires: Universidad de Palermo.

FreIRE, P. (1974). La educación como práctica de la libertad ( $15^{a}$ ed.). Buenos Aires: Siglo XXI editores.

Celorio, G. y López de Munain, A. (Coord.) (2007). Diccionario de Educación para el desarrollo. Bilbao: Hegoa. Recuperado de http://libros.metabiblioteca.org:8080/ ispui/bitstream/001/491/1/diccionario_2.pdf

LECHNER, N. (1983/2013). "Los derechos humanos como categoría política". En I. Semo, F. Valdés Ugalde y P. GutiérRez (Eds.), Obras II: ¿̨Qué significa hacer política?/ Norbert Lechner (pp. 247-257). México: Fondo de Cultura Económica-FLACSO.

- (1990). Los patios interiores de la democracia: subjetividad y política (2a ed.). Santiago de Chile: Fondo de Cultura Económica.

- (1991/2015). "Las ciencias sociales en el proceso de democratización". En I. SEMO, F. Valdés Ugalde y P. GutiérRez (Eds.), Obras IV: política y subjetividad, 1995-2003/ Norbert Lechner (pp. 27-33). México: Fondo de Cultura Económica-FLACSO.

Manzo Rodríguez, M., Rivera Michelena, C. y Rodríguez Orozco, A. (2006). "La educación de posgrado y su repercusión en la formación del profesional iberoamericano". Educación Médica Superior, 20(3). Recuperado de http://scielo. sld.cu/scielo.php?pid=S0864-21412006000300009\&script=sci_arttext\&tlng=pt

MejíA Jiménez, M. y MANJARRÉs, M. E. (2011). "La investigación como estrategia pedagógica". Praxis y Saber, 2(4), 127-177. Recuperado de https://www.redalyc. org/pdf/4772/477248388007.pdf 
MIRO, J. (201 1). "Repositorio de actividades para enseñar competencias transversales". Revista de Docencia Universitaria, 8(1) 101-110. Recuperado de https:// riunet.upv.es/bitstream/handle/10251/141819/Mir\%C3\%B3? sequence=1

NUÑEZ, M. (2012). "Una aproximación desde la sociología fenomenológica de Alfred Schütz a las transformaciones de la experiencia de la alteridad en las sociedades contemporáneas". Sociológica, año 27(75), ene./abr, 49-67. Recuperado de http://www.scielo.org.mx/pdf/soc/v27n75/v27n75a2.pdf

Nussbaum, M. (2012). Crear Capacidades. Propuesta para el desarrollo humano. Barcelona: Paidós.

ORTIZ, R. (2004). Taquigrafiando lo social. Buenos Aires: Siglo XXI editores.

PerRenOud, P. (2001). "La clave de los campos sociales: competencias del actor autónomo". En D. S. RYCHEN Y L. HERSH SALGANIK, Definiry seleccionar las competencias fundamentales para la vida (pp. 216-250). México: Fondo de Cultura Económica. Recuperado de http://rubenama.com/articulos/Perrenoud_campos_sociales.pdf

- (2004). Diez nuevas competencias para enseñar. Barcelona: Graó.

- (2008). "Construir las competencias, ¿̇es darle la espalda a los saberes?" REDU. Revista de Docencia Universitaria, núm. Monograf, II, 1-8.

Reiseberg, L. y Watson, D. (2011). "Acceso y equidad". En P. Altbach (Ed.), Liderazgo para universidades de clase mundial: desafíos para países en desarrollo (pp. 337-368). Cátedra UNESCO-ONU "Historia y futuro de la Universidad", Colección de Educación Superior. Buenos Aires: Universidad de Palermo.

Ruiz Olabuenaga, J. e Ispizua, M. (1989). La descodificación de la vida cotidiana: métodos de investigación cualitativa. Bilbao: Universidad de Deusto.

Schutz, A. (1973). El problema de la realidad social. Barcelona: Amorrortu.

SeN, A. (1998). "Las teorías del desarrollo en el Siglo XXI". En L. EMMERIJ y J. NúÑ̃EZ, (Comp.), El desarrollo económico y social en los umbrales del siglo XXI (pp. 75-100). Washington D.C.: Banco Interamericano de Desarrollo.

- (2000). Desarrollo y Libertad. Bogotá: Editorial Planeta. 
- (2002). "¿Por qué la equidad en salud?" Texto del discurso leído en la III Conferencia Internacional sobre Economía de la Salud. York, Reino Unido, 23 de julio de 2002. Revista Panamericana de Salud Pública, (5/6), 302-309. Recuperado de https://www.scielosp.org/pdf/rpsp/2002.v1 ln5-6/302-309/es

- (201 1). La idea de justicia. Buenos Aires: Taurus.

Sotolongo Codina, P. y Delgado Díaz, C. (2006). La revolución contemporánea del saber y la complejidad social. Buenos Aires: CLACSO.

\section{Otros documentos consultados}

Organización de las Naciones UnIDAs (ONU). Asamblea General. Resolución número 70/1. Transformando nuestro mundo: la Agenda 2030 para el Desarrollo Sostenible. Seguimiento de los resultados de la Cumbre del Milenio. Aprobada el 25 de septiembre de 2015.

Organización de las Naciones Unidas para la Educación, la Ciencia y la Cultura (2015). Replantear la educación: ¿̇Hacia un bien común mundial? París: Ediciones UNESCO. Recuperado de https://unesdoc.unesco.org/ark:/48223/pf0000232697 